\title{
Risk Factors for Falls in Patients with Alzheimer Disease: A Retrospective Study of Balance, Cognition, and Visuospatial Ability
}

\author{
Mayuka Okia Miyuki Matsumoto ${ }^{b}$ Yukiko Yoshikawab Mitsuko Fukushimab

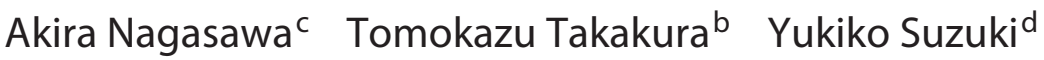 \\ ${ }^{a}$ Department of Rehabilitation Medicine, Juntendo University Hospital, Tokyo, Japan; ${ }^{\mathrm{b}}$ Department of Rehabilitation \\ Medicine, Juntendo Tokyo Koto Geriatric Medical Center, Koto, Japan; ' Department of Rehabilitation Medicine, \\ Juntendo University Koshigaya Hospital, Koshigaya, Japan; ${ }^{d}$ Department of Occupational Therapy, Kyorin \\ University, Mitaka, Japan
}

\section{Keywords}

Alzheimer disease $\cdot$ Fall · Functional reach test $\cdot$ Cutoff

\begin{abstract}
Aim: This study aimed to determine the main risk factors for falls in patients with Alzheimer disease (AD) by comparing balance, cognition, and visuospatial ability between those who have experienced a fall and those who have not. Methods: Forty-seven $A D$ patients were admitted to a ward for patients with dementia ( 22 men and 25 women). The balance of patients was evaluated using the Functional Reach Test (FRT), the one-leg standing duration, and the Timed Up and Go (TUG) test. The Mini-Mental State Examination-Japanese (MMSE-J) was used to evaluate cognition. For visuospatial ability assessment, the Clock-Drawing Test (CDT) as well as overlapping figure identification and shape discrimination in the Visual Perception Test for Agnosia (VPTA) were used. The patients were allocated to either the fall group or the nonfall group based on their history of falls in the past year. The relationships between patients' characteristics and evaluation outcomes were compared and examined. Logis-
\end{abstract}

tic regression analysis was performed using a fall as the objective variable. The area under the curve (AUC) and the cutoff value were calculated. Results: Of the 47 participants, 22 had experienced falls within the past year (46.8\%). The results of the FRT, one-leg standing duration, the TUG, the CDT, and the VPTA were significantly lower in the fall group. No significant difference between the MMSE-J scores of the fall group and those of the nonfall group was observed. The results of the logistic regression analysis indicated that falls in $A D$ patients were significantly associated with the FRT. It was found that a shorter FRT distance $(\mathrm{cm})$ had a significant impact on falls. For the FRT, the fall-related AUC was 0.755. At a cutoff value of $24.5 \mathrm{~cm}$, the level of sensitivity was $68.0 \%$, and the level of specificity was $77.3 \%$. Conclusions: The findings of this study indicate that balance and visuospatial abilities are risks factors for falls in AD patients. In contrast, cognitive impairment was not a risk factor for falls. It was demonstrated that the FRT could be an appropriate risk predictor for falls in $A D$ patients. In particular, falls in AD patients were strongly affected by a reduced dynamic balance.

(c) 2021 The Author(s)

Published by S. Karger AG, Basel karger@karger.com www.karger.com/dee

Karger $\stackrel{\text { ' }}{5}$

BOPEN ACCESS
(C) 2021 The Author(s)

Published by S. Karger AG, Basel

This article is licensed under the Creative Commons AttributionNonCommercial-NoDerivatives 4.0 International License (CC BYNC-ND) (http://www.karger.com/Services/OpenAccessLicense) Usage and distribution for commercial purposes as well as any distribution of modified material requires written permission.
Yukiko Suzuki

Department of Occupational Therapy, Kyorin University

Shimorenjyaku 5-4-1

Mitaka, Tokyo 181-8612 (Japan)

y-suzuki@ks.kyorin-u.ac.jp 


\section{Introduction}

A fracture from a fall accounts for approximately $10 \%$ of all causes that lead to long-term care needs in older adults. Therefore, falls in older adults are a serious concern for the community in Japan. Particularly, patients with dementia are at high risk for a fall [1-5]. It has been found that a patient can become bedridden following a fall, accelerating the progression of dementia [6]. Hence, prevention of falls in patients with dementia is key to averting the progression of dementia and the increased burden of nursing care. Studies have found that the causes of falls in patients with dementia include reduced balance $[7,8]$, advanced dementia [9], the presence of advanced white matter lesions as determined by a head MRI [10], behavioral and psychological symptoms of dementia [11], consumption of antipsychotics [10], hypertension [2], and a reduced visuospatial ability $[12,13]$. A few of these studies have reported on the relationship between a reduced visuospatial ability and falls and pointed out that, compared to gait disorders, a reduced visuospatial ability is more significantly associated with falls $[12,13]$. These studies have examined patients with dementia other than Alzheimer disease (AD). Therefore, they may not have sufficiently examined reduced balance in comparison with visuospatial disability in association with the risk of falls in patients with dementia. Hence, the authors decided to investigate which conditions among balance evaluation, cognition evaluation, or visuospatial ability evaluation best predict falls in patients with $\mathrm{AD}$. This study examined patients with $\mathrm{AD}$, which reportedly accounts for half to 3 quarters of the total cases of dementia [14]. We aimed to analyze the characteristics of falls in patients with AD by evaluating and comparing balance, cognition, and visuospatial ability between those who have experienced a fall and those who have not.

\section{Materials and Methods}

\section{Participants}

Forty-seven $\mathrm{AD}$ patients admitted to a ward for patients with dementia ( 22 men and 25 women; mean age $80.9 \pm 6.3$ years) were enrolled.

Patients were admitted to the dementia care ward for various purposes including: early diagnosis and early treatment of dementia, treatment of physical complications, and management of behavioral and psychological symptoms. The inclusion criteria were: consecutive admission to a dementia care ward and a diagnosis of $\mathrm{AD}$ by a physician based on the 10th version of the International Classification of Diseases (ICD-10). The exclusion criteria included the following 6 potential causes of falls: (1) inability to walk
$50 \mathrm{~m}$ or more (a functional independence measure [FIM] score of 5 points or lower for walking; (2) motor paralysis; (3) severe sensory disturbances; (4) a history of impaired consciousness, loss of consciousness, or delirium; (5) marked visual impairment; and (6) being considered ineligible for this study by the principal investigator. One of the authors evaluated each participant within 1 week of admission. Data on the participant's characteristics (sex, age, duration of $\mathrm{AD}$, number of years of education after graduating elementary school, comorbidities, number of types of medicines) were obtained from the hospital's medical records (Table 1).

\section{Evaluation}

Patients were evaluated using the following tests: the MiniMental State Examination-Japanese (MMSE-J) [15] for cognition; the Functional Reach Test (FRT) [16], one-leg standing duration, and the Timed Up and Go Test (TUG) [17] for balance; the ClockDrawing Test (CDT) [18] and overlapping figure identification and shape discrimination in the Visual Perception Test for Agnosia (VPTA) were sued to assess visuospatial ability.

This study depicted the method reported by Duncan et al. [16] for performing the FRT. Participants started the test in a standing position with their legs spread shoulder width apart. They then reached forward without changing the base of support, with their shoulder joints flexed at 90 degrees, elbow joints fully extended, and forearms pronated, which was the starting position of the limbs. In this position, the participant made a loose fist, and the tips of the middle fingers were set as the starting points. The horizontal distance between the starting and end points, which were the points that the participant could reach in the above position, were measured in centimeters using a measuring tape. The distance was measured twice. The higher measurement was used as the representative value.

For measurement of the one-leg standing durations, the participants rounded their shoulders and positioned their arms in front of the chest while keeping their eyes open. The duration for which each participant could maintain their posture, from the time when the sole of one of the feet leaves the floor to the time when it again touches the ground, was measured using a digital stop watch. The duration was measured twice on each side (right and left). The mean of the longer duration (in s) on both sides was used as the representative value.

The method of Okaji and Iida [17] was adopted for performance of the TUG. The time (in s) that it took for each participant to complete the following movements was measured: standing up from the chair, walking $3 \mathrm{~m}$ to the target object, turning around, walking back to the chair, and sitting down on it. Each participant started the test in a seated position with their back and buttocks in contact with the seat back. The measurement was started when the examiner said "go." The time from the start of measurement until the buttocks of the participant came in contact with the seat was measured using a digital stop watch.

The CDT evaluates visuospatial ability and executive function. It is a simple test that is widely used in dementia screening. In this study, the participants were instructed to draw a clock indicating 11:10 on white paper. Their performance was evaluated with a score ranging from 0 to 10 points in accordance with the method of Rouleau et al. [18]. The higher the score, the more favorable the participant's function.

For the overlapping figure identification and shape discrimination tasks of the VPTA, the participants were shown a picture of 
Table 1. Participants' characteristics and evaluation outcomes

\begin{tabular}{|c|c|c|c|c|c|}
\hline & $\begin{array}{l}\text { Total } \\
(n=47)\end{array}$ & $\begin{array}{l}\text { Fall group } \\
(n=22)\end{array}$ & $\begin{array}{l}\text { Nonfall group } \\
(n=25)\end{array}$ & $p$ value & $\begin{array}{l}\text { Effect } \\
\text { size }\end{array}$ \\
\hline Men/women ratio & $22 / 25$ & $7 / 15$ & $15 / 10$ & 0.080 & 0.28 \\
\hline Age, years & $80.9 \pm 6.3$ & $82.9 \pm 6.1$ & $79.1 \pm 6.0$ & $0.035^{*}$ & 0.31 \\
\hline Duration of $\mathrm{AD}$, months & $30(1-144)$ & $18(1-132)$ & $36(3-144)$ & 0.331 & 0.14 \\
\hline Education, years & $6(0-10)$ & $6(0-10)$ & $6(3-10)$ & 0.385 & 0.13 \\
\hline Comorbidities, $n$ & $5(1-12)$ & $5(1-11)$ & $5(1-12)$ & 0.932 & 0.01 \\
\hline Types of oral medicines, $n$ & $7(0-35)$ & $7(0-35)$ & $6(1-27)$ & 0.601 & 0.08 \\
\hline MMSE-J score ${ }^{a}$ & $16.4 \pm 5.4$ & $16.7 \pm 4.6$ & $16.2 \pm 6.1$ & 0.754 & 0.05 \\
\hline NPI score $\mathrm{b}^{\mathrm{b}}$ & $6(0-40)$ & $6(0-36)$ & $4(0-40)$ & 0.599 & 0.08 \\
\hline FIM score ${ }^{c}$ & $98(59-120)$ & $98(59-117)$ & $98(73-120)$ & 0.529 & 0.09 \\
\hline FRT, $\mathrm{cm}$ & $23.0 \pm 8.3$ & $19.5 \pm 7.0$ & $26.1 \pm 8.3$ & $0.005^{* *}$ & 0.40 \\
\hline One-leg standing duration, s & $4.2(0-91.8)$ & $2.5(0-5.8)$ & $8.0(0.8-91.8)$ & $0.000^{* *}$ & 0.55 \\
\hline TUG, s & $12.5(5.9-91.7)$ & $13.8(8.0-91.7)$ & $10.0(5.9-25.9)$ & $0.004^{* *}$ & 0.43 \\
\hline CDT score ${ }^{\mathrm{d}}$ & $5(0-10)$ & $3(0-10)$ & $6(0-10)$ & $0.021^{*}$ & 0.34 \\
\hline VPTA score $e^{e}$ & $0(0-5)$ & $1(0-5)$ & $0(0-5)$ & $0.011^{*}$ & 0.37 \\
\hline
\end{tabular}

Values are presented as means \pm SD or medians (range) unless otherwise stated. ${ }^{*} p<0.05 . * * p<0.01$. Sex: Fisher exact test. Age, MMSE-J, FRT: 2 -sample $t$ test. Other items: Mann-Whitney U test. ${ }^{a}$ Range: $0-30$ points; the higher the score, the more favorable the participant's function. ${ }^{b}$ Range: $1-120$ points; the higher the score, the worse the participant's symptoms. ${ }^{c}$ Range: $18-126$ points; the higher the score, the higher the degree of independence. ${ }^{\mathrm{d}}$ Range: $0-10$ points; the higher the score, the more favorable the participant's function. ${ }^{\mathrm{e}}$ Range: $0-6$ points; the lower the score, the more favorable the participant's function.

overlapping objects or geometric shapes on a card (card 1). They were asked to say the names of the objects or the number of the geometric shapes drawn. The participant was asked to trace the contour of each object and shape with a finger if they found it difficult to say the names of the objects or the number of the shapes. Subsequently, they were shown another card (card 2) on which multiple figures including those drawn on the previous card (card 1). The participants were shown both cards (cards 1 and 2) simultaneously. The participants were then asked to point to the shapes that were drawn on both cards. Their performance was evaluated with a score ranging from 0 to 6 points, which was based on the number of correct answers. The lower the score, the more favorable the participant's function.

In addition to the above evaluations, the Neuropsychiatric Inventory (NPI) was used to evaluate the behavioral and psychological symptoms of dementia, and the FIM was used to evaluate activities of daily living.

\section{Procedures}

All of the evaluations were performed by a physical therapist (with 14 years of experience) or an occupational therapist (with 10-16 years of experience) within a week after hospitalization. During all of the evaluation periods, the participants' oral medications and conditions remained unchanged.

Falls were defined as "any unintentional action where a part of the body other than the soles of the feet comes in contact with the ground or lower location" [19]. Information regarding the patient's fall history was obtained from the main caregiver. The main caregiver was also familiar with the patient's prehospitalization living conditions and therefore also provided information regard- ing the patient's prehospital history in the last year, for assessment.

The participants were categorized into those who had experienced falls within the last year (fall group; $n=22$ ) and those who had not (nonfall group; $n=25$ ).

\section{Data Analysis}

In comparing the fall group and the nonfall group, the Fisher exact test, a 2-sample $t$ test, and the Mann-Whitney $U$ test were used. The effect size $(\varphi)$ was calculated for the Fisher exact test, and the effect size $(r)$ was calculated for the 2-sample $t$ test and the Mann-Whitney U test. The index proposed by Cohen [20] was referenced to interpret the effect size, with $0.1-0.29$ as low, 0.30.49 as moderate, and $\geq 0.5$ as high.

Also, to validate the degrees of impacts, a logistic regression analysis (forward stepwise selection, the likelihood ratio test) was performed. In doing so, history of falls was set as an objective variable, and items for which significant differences were observed were set as explanatory variables.

Additionally, to confirm the effectiveness of fall prediction, a receiver operating characteristic (ROC) curve was obtained. In doing so, the results of the logistic regression analysis were set as independent variables, and history of falls as an objective variable. The cutoff value, the levels of sensitivity and specificity, and the area under the curve (AUC) were then calculated. The AUC varies between 0.5 and 1. An AUC $\geq 0.9$ indicates a high accuracy one between 0.7 and 0.9 indicates a medium accuracy, and one between 0.5 and 0.7 indicates a low accuracy [21].

SPSS (version 21.0, IBM Corporation, Armonk, USA) was used for the statistical analysis, and the significance level was set as $<5 \%$.
60 Dement Geriatr Cogn Disord Extra 2021;11:58-63 DOI: $10.1159 / 000514285$
Oki/Matsumoto/Yoshikawa/Fukushima/ Nagasawa/Takakura/Suzuki 
Table 2. Logistic regression analysis to identify factors associated with falls in $\mathrm{AD}$ patients admitted to a ward for patients with dementia

\begin{tabular}{llll}
\hline & OR & $95 \%$ CI & $p$ value \\
\hline FRT & 1.118 & $1.026-1.219$ & $0.011^{*}$ \\
\hline
\end{tabular}

The total number of patients is 47 . $^{*} p<0.05$. Dependent variables: fall group, nonfall group. Independent variables: 6 items, i.e., age, FRT, TUG, one-leg standing duration, CDT, and VPTA.

\section{Results}

The participant's characteristics are shown in Table 1. Of the 47 participants, 22 had fallen within the past year (46.8\%). The differences in sex, AD duration, number of years of education, number of comorbidities, types of oral medicines, NPI, and FIM were not significant between the fall and nonfall groups. The difference in age was significant. The fall group $(n=22)$ was significantly older than the nonfall group $(n=25)$.

Upon comparing cognition, balance, and visuospatial ability, the difference in FRT, one-leg standing duration, TUG, CDT, and VPTA was significant, with the fall group $(n=22)$ showing a significantly poorer result compared to that of the nonfall group $(n=25)$. Conversely, the difference in MMSE-J was not significant between the fall and nonfall groups (Table 1).

The 2 groups to which the participants were allocated based on their history of falls were set as objective variables. Items for which significant intergroup differences $(p<0.05)$ were observed were selected as explanatory variables. The possibility of multicollinearity was also examined. It was determined that there was no collinearity between the explanatory variables because the correlation coefficients in internal correlations between the variables, which were examined prior to the analysis, were 0.800 or below. Hence, binomial logistic regression analysis (forward stepwise selection, likelihood ratio test) was applied in choosing the optimal model. In doing so, the following 6 items were set as explanatory variables: age, FRT, oneleg standing duration, TUG, CDT, and the overlapping figure identification and shape discrimination tasks of the VPTA. The results indicated that falls in patients with AD were significantly associated with the FRT $(\mathrm{OR}=1.118$; 95\% CI 1.026-1.219). It was found that shorter FRT distances had a significant impact on falls (Table 2).

Based on the results of the logistic regression analysis, an ROC curve for the FRT, which was extracted as an

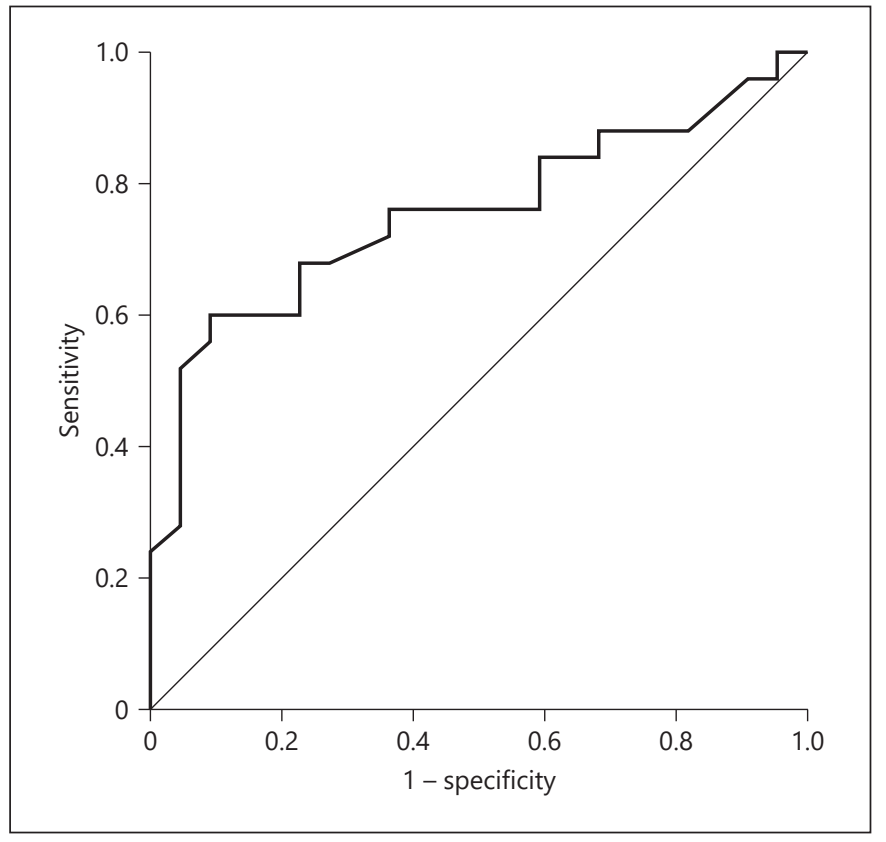

Fig. 1. At a cutoff value of $24.5 \mathrm{~cm}$ for the FRT, the level of sensitivity was $68.0 \%$ and the level of specificity was $77.3 \%$.

evaluation that had a strong association with falls, was created. The levels of sensitivity and specificity, and the cutoff value, were then examined. It was found that the fall-related AUC for the FRT was 0.755 (95\% CI 0.6130.896; $p=0.003$ ), and that the FRT had a moderate level of prediction precision as per the Anthony classification [21]. At a cutoff value of $24.5 \mathrm{~cm}$ for the FRT, the level of sensitivity was $68.0 \%$ and the level of specificity was $77.3 \%$ (Fig. 1).

\section{Discussion}

In this study, patients with $\mathrm{AD}$ were allocated to either the fall group or the nonfall group. The study then examined the differences between groups in terms of balance, cognition, and visuospatial ability. The results indicated between-group differences in the following evaluations: the FRT, one-leg standing duration, the TUG, the CDT, and the VPTA. The effect size $(r)$ for one-leg standing duration was found to be high at 0.55 . Moderate effect sizes were observed for other indicators $(r=0.34-0.43)$. These results indicated that balance and visuospatial ability are risk factors for falls in patients with $\mathrm{AD}$. van Dijk et al. [7] and Sterke et al. [8] both reported that a reduced balance is a predictor of falls. The results of this study 
concurred with their findings and confirmed that a reduced balance was associated with an increased risk of falls. A reduced visuospatial ability is reportedly a factor associated with falls in patients with dementia $[12,22]$. The results of this study support the findings from earlier studies. Falls among patients with AD are greatly affected by visuospatial ability, which can be evaluated by the CDT and the VPTA.

While a low CDT score was found to be a risk factor for falls, no significant differences in the MMSE-J were observed between the fall and nonfall groups. The MMSE-J evaluates general cognition including memory, orientation, attention, and spatial cognition. Meanwhile, the CDT evaluates not only visuospatial ability but also a combination of visuospatial and executive functions. Muir et al. [9] and Ambrose et al. [23] pointed out that a reduction in attention and executive functions (areas of cognition) is highly likely to increase the risk of falls. For an individual to not fall when they move, it is particularly necessary for them to exert executive functions such as controlling their thoughts and behaviors. This may explain why the incidence of falls is greatly affected by executive functions, which can be evaluated by the CDT.

In this study, we used multiple indicators to evaluate balance and visuospatial ability. Through these evaluations, the study validated the degree of impact of these indicators on falls in patients with AD. Based on the results of the logistic regression analysis, the FRT was extracted as an indicator that is strongly associated with the risk of falls. The ROC curve indicated that the fallrelated AUC was 0.755 , suggesting that the FRT has a moderate level of prediction precision as per the Anthony classification [22]. These results indicated that poor FRT results are associated with an increased risk of falls. The FRT measures the distance that the participants can reach when they stretch their arms forward in a standing position. It evaluates dynamic balance in a standing position. The results of this study indicated that a patient with $\mathrm{AD}$ who has a reduced dynamic balance has a higher likelihood of falling. Clinical evaluations that are time consuming to complete and those with a high level of difficulty may need to be suspended mid-evaluation if the patient who is being evaluated complains of fatigue or refuses to continue. When evaluating a patient with $\mathrm{AD}$, it is necessary for the therapist to collect accurate information on the characteristics of the patient by using a small number of simple indicators. The FRT is a good test, as it takes only several minutes to perform. Also, only simple in- structions are needed to perform the test. Therefore, FRT results can be used as an effective clinical predictor of falls in patients with $\mathrm{AD}$.

The ROC curve indicated that the levels of sensitivity and specificity were 68.0 and $77.3 \%$, respectively, when the FRT result was $24.5 \mathrm{~cm}$. Several studies have reported on FRT cutoff values for falls in patients with different diseases. Thomas and Lane [24] reported that an FRT result $<18.5 \mathrm{~cm}$ was the cutoff value that could be used as a criterion for the risk of falls in fragile older adults. Another study reported that an FRT result $<15.0$ $\mathrm{cm}$ could be a criterion for the risk of falls in patients with poststroke hemiplegia [25]. Yet another study reported that an FRT result of $31.75 \mathrm{~cm}$ was the cutoff value for falls in Parkinson disease [26]. As shown above, various studies have examined the FRT as a predictor of falls. The results of this study indicated that the cutoff value for falls in patients with $\mathrm{AD}$ was an FRT result of $24.5 \mathrm{~cm}$, meaning that a patient with an FRT result $<24.5 \mathrm{~cm}$ is at risk of falls. A therapist may use FRT results as an indicator for falls in patients with AD. In other words, a therapist may be able to select those at risk of falls using the FRT and provide advanced care to prevent falls for a patient with AD whose FRT result is $24.5 \mathrm{~cm}$ or shorter, including a fall prevention program and reminding the patient to take every precaution to prevent falls.

One of the limitations of this study is that evaluation by the CDT may include factors other than visuospatial ability. This is because the CDT usually measures not only visuospatial ability but also a combination of visuospatial and executive functions. This study focused only on patients with $\mathrm{AD}$ admitted to a dementia care ward, and hence generalization of the results may not be possible. To obtain more generalizable results, patients with $\mathrm{AD}$, who are living at home, should also be included in the investigation of associated factors and history of falls. Moreover, it should be noted that this study retrospectively examined history of falls in a small number of cases. Future studies are warranted to prospectively examine factors for falls in a larger number of patients.

\section{Acknowledgement}

We express our sincere gratitude to all the staff members of the Juntendo Tokyo Koto Geriatric Medical Center, Juntendo University, as well as all of the participants and their families, for their cooperation with this study.
62

Dement Geriatr Cogn Disord Extra 2021;11:58-63 DOI: $10.1159 / 000514285$
Oki/Matsumoto/Yoshikawa/Fukushima/ Nagasawa/Takakura/Suzuki 


\section{Statement of Ethics}

This study was approved by the Institutional Review Board of the Juntendo Tokyo Koto Geriatric Medical Center, Juntendo University Hospital (approval No. 96-4) and Faculty of Health Sciences, Kyorin University (approval No. 29-64). The aims of this study were explained verbally and in writing to the participants and relatives who lived with the participants, and they provided written informed consent.

\section{Conflict of Interest Statement}

The authors have no conflict of interests to declare.

\section{Funding Sources}

The authors have no funding sources to declare.

\section{Author Contributions}

M.O. designed this study, collected the data, interpreted the results, and wrote this paper. M.M. collected the data, searched the literature, and wrote this paper. Y.Y. and M.F. collected the data. A.N. and T.T. contributed to the writing of this paper. Y.S. designed this study, performed the statistical analysis, interpreted the results, and wrote this paper. All of the authors approved this paper for submission. The first author (M.O.) died on June 10, 2020. M.O. contributed as much to the writing of this paper as the second author (M.M.). M.O. died after reviewing this paper (in Japanese).

\section{References}

1 Buchner DM, Larson EB. Falls and fractures in patients with Alzheimer-type dementia. JAMA. 1987 Mar;257(11):1492-5.

2 Morris JC, Rubin EH, Morris EJ, Mandel SA. Senile dementia of the Alzheimer's type: an important risk factor for serious falls. J Gerontol. 1987 Jul;42(4):412-7.

3 Asada T, Kariya T, Kinoshita T, Asaka A, Morikawa S, Yoshioka M, et al. Predictors of fall-related injuries among communitydwelling elderly people with dementia. Age Ageing. 1996 Jan;25(1):22-8.

4 van Doorn C, Gruber-Baldini AL, Zimmerman S, Hebel JR, Port CL, Baumgarten M, et al.; Epidemiology of Dementia in Nursing Homes Research Group. Dementia as a risk factor for falls and fall injuries among nursing home residents. J Am Geriatr Soc. 2003 Sep; 51(9):1213-8.

5 Allan LM, Ballard CG, Rowan EN, Kenny RA. Incidence and prediction of falls in dementia: a prospective study in older people. PLoS One. 2009;4(5):e5521.

6 Yukimasa T, Nakano M, Matsuura $H$, Tamagawa M, Yamanaka M, Kubota M. The investigation of risk factor of injury caused by falling [in Japanese]. J Biomed Fuzzy Sys Assoc. 2011;13:109-14.

7 van Dijk PT, Meulenberg OG, van de Sande HJ, Habbema JD. Falls in dementia patients. Gerontologist. 1993 Apr;33(2):200-4.

8 Sterke CS, Huisman SL, van Beeck EF, Looman CW, van der Cammen TJ. Is the Tinetti Performance Oriented Mobility Assessment (POMA) a feasible and valid predictor of short-term fall risk in nursing home residents with dementia? Int Psychogeriatr. 2010 Mar;22(2):254-63.
9 Muir SW, Gopaul K, Montero Odasso MM. The role of cognitive impairment in fall risk among older adults: a systematic review and meta-analysis. Age Ageing. 2012 May;41(3): 299-308.

10 Horikawa E, Matsui T, Arai H, Seki T, Iwasaki K, Sasaki H. Risk of falls in Alzheimer's disease: a prospective study. Intern Med. 2005 Jul;44(7):717-21.

11 Suzuki M, Kurata S, Yamamoto E, Makino K, Kanamori M. Impact of fall-related behaviors as risk factors for falls among the elderly patients with dementia in a geriatric facility in Japan. Am J Alzheimers Dis Other Demen. 2012 Sep;27(6):439-46.

12 Olsson RH Jr, Wambold S, Brock B, Waugh $D$, Sprague H. Visual spatial abilities and fall risk: an assessment tool for individuals with dementia. J Gerontol Nurs. 2005 Sep;31(9): 45-51.

13 Eriksson S, Gustafson Y, Lundin-Olsson L. Characteristics associated with falls in patients with dementia in a psychogeriatric ward. Aging Clin Exp Res. 2007 Apr;19(2): 97-103.

14 Diaz Brinton R, Yamazaki RS. Advances and challenges in the prevention and treatment of Alzheimer's disease. Pharm Res. 1998 Mar; 15(3):386-98.

15 Mori E, Mitani Y, Yamadori A. Usefulness of a Japanese version of the test in neurological patients [in Japanese]. Japanese J Neuropsychol. 1985;1:82-90.
16 Duncan PW, Weiner DK, Chandler J, Studenski S. Functional reach: a new clinical measure of balance. J Gerontol. 1990 Nov;45(6):M1927.

17 Okaji T, Iida H. Timed Up \& Go Test [in Japanese]. J Phys Ther. 2005;22:129-36.

18 Rouleau I, Salmon DP, Butters N, Kennedy C, McGuire K. Quantitative and qualitative analyses of clock drawings in Alzheimer's and Huntington's disease. Brain Cogn. 1992 Jan; 18(1):70-87.

19 Kondo S, Miyamae T, Tsutsumi F. Fear of falling among elderly persons [in Japanese]. General Rehabilitation. 1999;27:775-80.

20 Cohen J. A power primer. Psychol Bull. 1992 Jul;112(1):155-9.

21 Akobeng AK. Understanding diagnostic tests 3: receiver operating characteristic curves. Acta Paediatr. 2007 May;96(5):644-7.

22 Yamada M, Takechi H, Mori S, Aoyama T, Arai H. Global brain atrophy is associated with physical performance and the risk of falls in older adults with cognitive impairment. Geriatr Gerontol Int. 2013 Apr;13(2):437-42.

23 Ambrose AF, Cruz L, Paul G. Falls and Fractures: A systematic approach to screening and prevention. Maturitas. 2015 Sep;82(1):85-93.

24 Thomas JI, Lane JV. A pilot study to explore the predictive validity of 4 measures of falls risk in frail elderly patients. Arch Phys Med Rehabil. 2005 Aug;86(8):1636-40.

25 Acar M, Karatas GK. The effect of arm sling on balance in patients with hemiplegia. Gait Posture. 2010 Oct;32(4):641-4.

26 Dibble LE, Lange M. Predicting falls in individuals with Parkinson disease: a reconsideration of clinical balance measures. J Neurol Phys Ther. 2006 Jun;30(2):60-7. 